\title{
Studies on fixation by means of cultured cells
}

\author{
Mitio Niızima \\ Department of Anatomy Tokyo Medical and Dental University Medical School.
}

Although fixation occupies the most essential part of cytological and cytochemical procedures, its nature and mechanism remain still obscure. Most of researches concerning analysis of fixing effect of various kinds of fixatives have been carried out with sectioned preparations, so that the results obtained could not eliminate effects of other subsequent treatments such as washing, dehydration, infiltration with embedding materials and staining. In order to catch genuine morphological effects of fixation, it would be necessary to compare both states of cells before and after application of fixatives. For direct comparison of the structures of the same cells in the living state and after fixation, it would be suitable to employ tissue culture method. Many cytologists have examined separate living cells with the light-microscope and noticed the changes produced in them when a fixative was run below the cover-slip. Strangeway and Canty (1927) have done this kind of work by dark-ground microscopy. The invention of phase contrast microscope led to a series of similar studies.

Notwithstanding precise description of changes of the cellular structures is still lacking, because changes occuring on each individual structural component is difficult to catch by a simple observation or by taking still photomicrography, since a granule changes its location in an instant of action of a fixative, so that identification of, for example, each granule is almost impossible. Therefore, to observe and record the instantaneous changes of structures during application of fixatives, the author employed phase contrast cinematography combined with still photomicrography. Under a phase contrast microscope a cultured cell was observed in a perfusion chamber, photographed with ordinary photomicrography technique, and then, while running $16 \mathrm{~mm}$ movie-film with high sensitivity (ASA 250), various kinds of fixatives in liquid form were introduced into the chamber under continuous checking of optical focus of the microscope. A special type of perfusion chamber was made for this purpose.

Before application of fixatives the nutrient media of the culture were washed and replaced by Gey's balanced salt solutions in order to avoid possible hindrance of observation as a result of muddling of the nutrient medium. To follow the structural changes occuring during duration of fixation, the same cells were observed repeatedly at various intervals of time after exposure to the fixative. For simplicity of comparison chick fibroblastic cells were chosen as material and dark phase contrast objectives were employed.

Some of the results obtained will be given as follows:

(1) $10 \%$ Formol ( $\mathrm{pH} \mathrm{7.1)}$ 
First sign of action of the fixative is cessation of the movement of cytoplasmic granules. Then blisters appear on the cell-surface. A little later, light vacuoles come in sight in the cytoplasm. At the same time long filamentous mitochondria are broken up into fragments which swell into vesicular formation with a distinct wall. The cell also swells as a whole. No formation of new visible granules which were not discernible before fixation are seen. Lipid droplet comes in contact with neighbouring one keeping its original globular shape. Nuclear memebrane becomes distinct. Nucleoplasm becomes slightly turbid without any apparent granulation. Nucleolus appears dark as in the living state. As a whole it must be said that remarkable changes occur in the structure of cytoplasm although the latter preserves still transparency as in the living state.

(2) $19 \%$ Osmium tetroxide.

Almost no change of outer shape of the cell is seen. Cytoplasmic structures are preserved optically almost as in the living state, except for slight swelling of mitochondria, slight optical unequalization of cytoplasm as well as deformation of fat globules showing fine rumpling of its surface-membrane, which was noticed more precisely by electron-microscopy. On the contrary, the nuclear sap which appeared quite transparent before fixation becomes cloudy and the nucleolus loses its clear contrast.

(3) Formol-calcium.

On the surface of the cell gray vesicles appear soon after application of the fixative and grow gradually. While filamentous mitochondria are kept almost unchanged, fine granules become visible among them. By and by light transparent vesicles appear in the cytoplasm bringing about dislocation and drift of dark granules between them. Deformation of fat globules become discernible about one hour after initial introduction of the fixative.

(4) Glacial acetic acid.

Nuclear membrane becomes distinct. Nucleolus appears dark.

Granular formation in the nucleoplasm is remarkable, while in the cytoplasm it is rather weak and fine.

(5) Regaud's fluid.

Shape of the cell body is kept as in a living state. Mitochondria remain almost intact, but fine granulation in the cytoplasm makes them partly obscure.

(6) Zenker-formol.

Immediately after introduction of the fixative, sudden and marked change occures in the cytoplasm as well as in the nucleus. Fine and coarse dark granules appear newly in the cytoplasm with turbidity and swelling of the latter. Shape of mitochondria is preserved almost intact. Fat droplets show a tendency to stick to adjacent ones and increase their luster. Nucleolus becomes angular with increased optical density. Fine fibrils become discernible in the cytoplasm.

(7) Ciaccio's fluid.

Cytoplasm is filled with fine granules. Fat globules are seen as a brightly shining round structures surrounded by a dimly shining gray crescent. Mitochondria are completely destroyed. In the nucleus appear fine granules. Nuclear 
membrane becomes apparent.

(8) Susa.

One of the most abruptly acting fixative. Sudden coagulation and granuation appear in the cytoplasm as well as in the nucleus. Fine granulation of the nucleoplasm is a characteristic of this fixative. Fibrillar formation in the cytoplasm and sharpening of cytoplasmic processes are also characteristic. Mitochondria are destroyed.

(9) Cold aceton

Granules appearing after fixation in the cytoplasm are coarser than in the nucleus. Nuclear membrane is not so distinct, and fine granules are filled in the cytoplasm, while the nucleolus shines in a gray tone.

(10) Absolute ethyl alcohol.

The most remarkable characteristic of this fixative is shrinkage of the cell body occuring in an instant. Coarse granules appear also in the cyto- and nucleoplasm. Mitochondria are broken completly and fat globules disappear within a minute.

(11) Absolute methyl alcohol.

Immediately after invasion of the fixative, changes appear first in the nucleoplasm forming coarse granules, then finer granules are formed in the cytoplasm. Mitochondria disappear.

(12) Potassium permanganate. (3\%)

Immediately with perfusion of the fixative the visual field under the microscope becomes quite obscure, so that any occurence cannot be discernible. After washing with destilled water deformation of fat globules was seen : fungiform protrusion appears from a fat globule showing eruption of the covering membrane. Nucleus becomes obscure with diminished contrast of the nucleolus. Cytoplasmic structures lose their sharp configuration.

For the purpose of functional cytology fixation should mean at first preservation of the structures in the living state as far as possible. In this connection osmic acid appears to be the best fixative. Fixatives other than osmic acid cause more or less distorsion of cellular constituents. It should be also emphasized that changes which occur in the structure of a cell depends largely upon the state of cell life at the moment of fixation. A freshly living cell react vigorously against fixatives, so that marked changes result in the cell body. A dull cell, on the contrary, shows dull reaction with less remarkable changes in structure. So it may be rather good, in order to obtain good result of fixation, not to use too fresh material. This fact, however, needs further studies under different conditions. In addition, we must of course point out that fixation involves more than preserving cells in as life-like state as possible.

\section{Discussion}

Dr. Okuda: I wish to ask 1) the morphological change of mitochondria through formolcalcium and alcohol fixation 2) $\mathrm{pH}$ and temperature of formalin used.

Dr. Niizima : 
1) The characteristics of mitochondrial change through $10 \%$ formalin fixation are formation of foam like structure in mitochondria and fragmentation of long mitochondria.

2) Of $10 ? 0$ formalin, $\mathrm{pH}$ was 7.1 , and temperature was similar to that of animal.

3) The use of perfusion chamber for cinematography results in delayed action of fixatives and, therefore, the cinematographic findings did not show the ideal states of fixation.

\title{
Histochemical studies on the caudal neurosecretory system of the teleost fish, Cyprinus carpio
}

\author{
Y. Sano, M. Kawamoto and K. Hamana \\ (Anatomical Department, Kyoto Prefectural Medical College)
}

\begin{abstract}
Recently, the caudal neurosecretory system of the fish spinal cord has been found as a new one similar to the hypothalamic-hypophysial neurosecretory system in vertebrates and to the intercerbralis-cardiacum-allatum system in insects. All these are common in some organizations such as synthesis, transportation, storage and release of the secretory materials. There are, however, some differences in several staining reactions between the neurosecretory materials of the hypothalamic and of the spinal neurosecretory system. This is considered to be due to the difference in chemical composition of the secretory granules in these systems. Nothing has been known on the chemical composition and the biological activity of the hormone produced in the caudal neurosecretory system. For the purpose to solve some problems of this organ, histochemical observations of this system and of its secretory substances were caried out, comparing it with those of the other neurosecretory systems.
\end{abstract}

\section{Materials and methods}

Cyprinus carpio (100), of $20-42 \mathrm{~cm}$ in sizes and of $400-1200 \mathrm{gr}$. in weights, caught during winter and spring were used in this study. The caudal parts of their spinal cord were removed and fixed with abs. alcohol, 10\% formalin, Bodian's, Bouin's, Carnoy's, Helly's, Orth's solutions and Susa. Some of the materials were embedded in paraffin and sectioned at $6-8 \mu$. The other were cut at $20 \mu$ using freezing method.

Following stainings were used to inquire the some chemical properties of caudal neurosecretory system :

General: hematoxylin-eosin, one-step trichrome stain (GOMORI 1951), Azan, iron hematoxylin (HEIDENHAIN)

enzyme : lead phosphate method for acid phosphatase (GOMORI 1950), calsiumcobalt method for alkaline phosphatase (DEMPSEY \& DEANE 1946), tween method for lipase (GOMORI), neotetrazolium method for succinic dehydro- 